\title{
Effects of professional oral health care on reducing the risk of chemotherapy-induced oral mucositis
}

\author{
Hirokazu Saito • Yutaka Watanabe • Kazumichi Sato • \\ Hiroaki Ikawa • Yoshifumi Yoshida • Akira Katakura • \\ Shin Takayama $\cdot$ Michio Sato
}

Received: 18 December 2013 / Accepted: 6 May 2014 / Published online: 23 May 2014

(C) The Author(s) 2014. This article is published with open access at Springerlink.com

\begin{abstract}
Purpose Recent years have seen remarkable progress in cancer therapy, although treatment-induced adverse reactions and complications are not uncommon. Approximately $40 \%$ of patients undergoing chemotherapy for cancer experience adverse reactions in the oral cavity, with nearly half of them developing severe oral mucositis that necessitates postponing therapy and/or changing the drug dosage. The objective of this study was to assess the usefulness of prophylactic professional oral health care (POHC) for preventing mucositis in patients undergoing chemotherapy.

Methods Twenty-six female patients scheduled for chemotherapy for breast cancer were included in this study and randomized to the self-care or POHC groups. Assessment parameters included oral cavity photographs, plaque control records, Saxon test scores, Oral Assessment Guide scores, and grading using the Common Terminology Criteria for Adverse
\end{abstract}

H. Saito $\cdot$ Y. Yoshida $\cdot$ A. Katakura

Department of Oral Medicine, Oral and Maxillofacial Surgery, Tokyo

Dental College, 5-11-13 Sugano, Ichikawa, Chiba 272-8513, Japan

Y. Watanabe $(\square)$

Department of Oral Diseases Research, National Center for

Geriatrics and Gerontology, 35, Gengo, Morioka-machi, Obu-City,

Aichi 474-8511, Japan

e-mail: ywata@ncgg.go.jp

K. Sato

Oral Cancer Center, Tokyo Dental College, 5-11-13 Sugano,

Ichikawa, Chiba 272-8513, Japan

H. Ikawa

Research Center Hospital for Charged Particle Therapy, National Institute of Radiological Sciences, 4-9-1, Anagawa, Inage-ku, Chiba-shi, Chiba 263-8555, Japan

S. Takayama $\cdot$ M. Sato

Department of Surgery, Tokyo Dental College, 5-11-13 Sugano, Ichikawa, Chiba 272-8513, Japan
Events. Beginning before surgery and continuing through the completion of chemotherapy, the $\mathrm{POHC}$ patient group received weekly professional oral health care, including scaling, professional cleaning of the tooth surfaces, brushing instructions, and nutritional and lifestyle guidance.

Results More patients in the self-care group developed oral mucositis than in the POHC group. The Oral Assessment Guide score, which was used as an index of oral mucositis, was also significantly lower in the POHC group. Based on the Oral Assessment Guide and plaque control records, there was almost no deterioration of the oral environment in the POHC group, whereas deterioration was observed in the self-care group.

Conclusions These findings demonstrate the efficacy of regular POHC in reducing the risk of oral mucositis in breast cancer patients undergoing chemotherapy.

Keywords Oral health care $\cdot$ Breast cancer $\cdot$ Cancer chemotherapy · Oral mucositis · Randomized controlled study · Quality of life

\section{Introduction}

Recent years have seen remarkable progress in cancer therapy, although chemotherapy-induced adverse reactions and complications are not uncommon. For example, it has been reported that approximately $40 \%$ of patients undergoing chemotherapy for cancer develop adverse reactions in the oral cavity, with approximately half of the patients developing severe oral mucositis that necessitates changing, postponing, or discontinuing therapy [1-3]. Pain due to oral mucositis can make it difficult for patients to ingest food, potentially leading to malnutrition [4]. This can be accompanied by general health risks, including lowered immunity and aspiration of food due to swallowing disorders. Moreover, difficulty in 
ingesting food and poor oral hygiene can result in a synergistic increase in oral bacteria and an increased risk of aspiration pneumonia, resulting in a vicious circle.

A report from the USA demonstrated that the presence of oral/gastrointestinal mucositis symptoms increased the mortality rate due to infections by 4 -fold in patients who developed bone marrow suppression and mucositis and increased the risk of infection 2-fold in patients with bone marrow suppression during chemotherapy. A Physician Data Query from the National Cancer Institute revealed that the rapid metabolic cycle of oral mucosal cells leads to increased susceptibility to treatment-related toxicity, and secondary infection by complex microflora results in the exacerbation of mucositis symptoms [5].

In response to recently issued guidelines regarding oral mucositis, it is becoming common practice to include dental intervention as an essential component of cancer therapy [6]. In Japan, the Japan Dental Association and the National Cancer Center recommend preventing adverse dental events by means of dental care before and after surgery for cancer patients, thus improving the quality of life of patients undergoing treatment [7]. Moreover, Yoneyama et al. reported on the importance of professional oral care for the elderly [8].

Therefore, it can be expected that with increasing longevity and improved dental care in Japan, there will be a concomitant increase in the number of elderly patients with teeth remaining and/or complex oral prostheses who undergo chemotherapy. This scenario raises the possibility that the complex oral environment will be conducive to poor oral hygiene in patients undergoing chemotherapy, as well as exacerbation of oral cavity adverse reactions $[2,7]$. Furthermore, it has been postulated that improvement of oral health would alleviate the symptoms of oral mucositis in patients undergoing chemotherapy for solid tumors. Unfortunately, this hypothesis has not been validated [9]. To address this issue, we conducted the present study with the aim of assessing the efficacy of prophylactic professional oral health care (POHC), performed by dentists and dental hygienists, in reducing chemotherapyinduced oral mucositis in cancer patients.

\section{Patients and methods}

\section{Patients}

From July 2010 through September 2011, the Department of Surgery at Tokyo Dental College, Ichikawa General Hospital, gave oral and written explanations of the present study to patients who had been diagnosed with breast cancer and were scheduled to undergo chemotherapy. Twenty-six patients gave written informed consent to participate and were enrolled in the study. In Japan, the standard treatment for breast cancer is adjuvant chemotherapy on an outpatient basis. All participants in this study had stage I-IV disease according to the criteria of the Union for International Cancer Control, and they were scheduled to undergo adjuvant chemotherapy [10].

Most patients received a treatment regimen consisting of fluorouracil, epirubicin, and cyclophosphamide (fluorouracil $500 \mathrm{mg} / \mathrm{m}^{2}+$ epirubicin $100 \mathrm{mg} / \mathrm{m}^{2}+$ cyclophosphamide $500 \mathrm{mg} / \mathrm{m}^{2}$ ). For all patients, treatment was administered as outpatient neoadjuvant/adjuvant chemotherapy (Table 1). In addition, there was no significant difference in chemotherapy regimens between the $\mathrm{POHC}$ and self-care groups [5].

The exclusion criteria included absence of informed consent, difficulty in performing $\mathrm{POHC}$ due to cognitive impairment, or judgment by the investigator that the patient was not suitable for enrollment. The study was designed as a randomized controlled study, and the protocol was approved by the Ethics Committee of Tokyo Dental College, Ichikawa General Hospital (Approval Number 204).

\section{Methods}

The enrolled patients were randomly allocated to either the self-care or POHC group. At the time of the first chemotherapy administration, patients in the self-care group were given the usual interview discussing adverse oral reactions due to chemotherapy, and patients were assessed (see "Assessment parameters" section) and received brushing, nutrition, and lifestyle guidance. The oral health care approach applied to the POHC group was as follows. Prior to the start of chemotherapy and at the time of the first chemotherapy administration, the patients were interviewed in the usual manner, assessed (see "Assessment parameters" section), and given instructions on brushing, nutrition, and lifestyle. In addition, patients received weekly $\mathrm{POHC}$ consisting of scaling and polishing. During weekly POHC, the status of the oral cavity was determined, and further guidance was given on the basis of that determination. Reassessment was performed in the second week after chemotherapy initiation, which is believed to be the time when the symptoms of mucositis are most severe $[1,2]$. The interviews, assessments, and advice (and treatment in the POHC group) were repeated after the second cycle of chemotherapy in both groups.

In the self-care group, if the oral cavity exhibited symptoms of oral mucositis while the patient was on chemotherapy, POHC was instituted from that time on. For both patient groups, other dental therapies were performed before chemotherapy initiation, as deemed necessary on the basis of the initial assessments [2]. Continued instruction regarding nutrition and oral hygiene was given during the course of chemotherapy. In patients with more severe periodontitis, tooth extraction was performed as necessary.

To confirm that there were no differences in patient background characteristics between the two groups, patients were compared for age, body mass index (BMI), and number of 
Table 1 Regimen of adjuvant chemotherapy and deterioration rates for OAG and oral mucositis grade

\begin{tabular}{|c|c|c|c|c|c|c|}
\hline \multirow[t]{2}{*}{ Regimen } & \multicolumn{2}{|c|}{ Total subjects } & \multicolumn{2}{|c|}{ Patients with deterioration of OAG (\%) } & \multicolumn{2}{|c|}{ Patients with deterioration of oral mucositis grade $(\%)$} \\
\hline & Self-care & $\mathrm{POHC}$ & Self-care & $\mathrm{POHC}$ & Self-care & $\mathrm{POHC}$ \\
\hline FEC & 9 & 9 & $7(77.8)$ & $1(11.1)$ & $3(33.3)$ & $0(0.0)$ \\
\hline PTX & 4 & 1 & $2(50.0)$ & $0(0.0)$ & $1(25.0)$ & $0(0.0)$ \\
\hline DTX & 1 & 0 & $0(0.0)$ & $0(0.0)$ & $0(0.0)$ & $0(0.0)$ \\
\hline $\mathrm{TC}$ & 0 & 2 & $0(0.0)$ & $0(0.0)$ & $0(0.0)$ & $0(0.0)$ \\
\hline Total & 14 & 12 & $9(64.2)$ & $1(8.3)$ & $4(28.6)$ & $0(0.0)$ \\
\hline
\end{tabular}

FEC 5-fluorouracil $500 \mathrm{mg} / \mathrm{m}^{2}+$ epirubicin $100 \mathrm{mg} / \mathrm{m}^{2}+$ cyclophosphamide $600 \mathrm{mg} / \mathrm{m}^{2}, P T X$ paclitaxel $80 \mathrm{mg} / \mathrm{m}^{2}, D T X$ docetaxel $60 \mathrm{mg} / \mathrm{m}^{2}, T C$ docetaxel $70 \mathrm{mg} / \mathrm{m}^{2}+$ cyclophosphamide $600 \mathrm{mg} / \mathrm{m}^{2}$ ), $O A G$ Oral Assessment Guide, $P O H C$ professional oral health care

teeth. In addition, blood test values before chemotherapy and 2 weeks after chemotherapy were compared between both patient groups.

All dentists who participated in this study met the criteria established by the oral care team of Tokyo Dental College, Ichikawa General Hospital. Similarly, the dental hygienists who performed POHC in this study met the criteria established by the oral care team.

\section{Assessment parameters}

The assessment parameters consisted of oral cavity photographs, assessment of the oral mucositis grade using the United States National Cancer Institute Common Terminology Criteria for Adverse Events version 3.0 (NCI-CTCAE) [11], evaluation of the oral environment using Eilers' Oral Assessment Guide (OAG) [12, 13], evaluation of oral hygiene using plaque control records (PCR) [13, 14], evaluation of dry mouth and oral moisture using the Saxon test $[15,16]$ and Mucus $^{\mathrm{TM}}[16,17]$, and gustatory measurements using an electrogustometer [18-22]. In addition, inflammation of the oral mucosa due to local or systemic factors, which may involve the buccal and labial mucosa, palate, tongue, floor of the mouth, and gingiva, was also assessed in this study.

Oral mucositis was graded according to the NCI-CTCAE criteria as follows: grade I, erythema of the mucosa; grade II, patchy ulcerations or pseudomembranes; and grade III, confluent ulcerations or pseudomembranes and bleeding with minor trauma [11].

\section{Statistical analysis}

For statistical analyses of the results, the POHC group was compared with the self-care group before chemotherapy and at 2 weeks after chemotherapy initiation, and the data for each assessment parameter were subjected to the Mann-Whitney $U$ test and the $\chi^{2}$ test. Statistical analyses were performed using SPSS version 19 statistical software (SPSS Inc., Chicago,
USA). $P$ values of $\leq 0.05$ were considered statistically significant.

\section{Results}

Patient background characteristics

A total of 26 patients with histopathologically confirmed breast adenocarcinoma were included in the study and randomized to the self-care group $(n=14)$ or the POHC group $(n=12)$. All patients were female, and the mean age was $55.8 \pm 9.4$ years. There were no significant differences between the two groups with regard to age, BMI, or number of teeth (Table 2).

No adverse dental events, such as acute dental infections, were observed during the course of this study. All patients completed chemotherapy, and no mortality was observed during the study period.

\section{Blood test values}

Hematological assessment demonstrated that postchemotherapy white blood cell counts, platelet counts, and levels of hemoglobin, total protein, and albumin were not significantly different from the respective pre-chemotherapy values in both groups (Table 3 ).

Table 2 Patient background characteristics

\begin{tabular}{llll}
\hline & Self-care $(n=14)$ & POHC $(n=12)$ & $P$ value \\
\hline Age & $58.4 \pm 9.8$ & $52.8 \pm 8.4$ & 0.128 \\
BMI & $22.4 \pm 3.3$ & $21.8 \pm 3.8$ & 0.655 \\
Number of teeth & $22.5 \pm 7.2$ & $22.7 \pm 7.7$ & 0.955 \\
\hline
\end{tabular}

POHC professional oral health care, $B M I$ body mass index 
Table 3 Blood test values before and after 2 weeks of chemotherapy (Mann-Whitney $U$ test)

\begin{tabular}{llccc}
\hline & & Self-care $(n=14)$ & POHC $(n=12)$ & $P$ value \\
\hline WBC & Before & $5,300.00 \pm 1,384.95$ & $6,300.00 \pm 1,550.66$ \\
& 2nd week after & $3,500.00 \pm 2,220.78$ & $4,950.00 \pm 2,160.58$ & 0.06 \\
PLT & Before & $26.25 \pm 4.79$ & $25.80 \pm 3.83$ & 0.237 \\
& 2nd week after & $32.40 \pm 10.80$ & $32.35 \pm 10.64$ & 0.877 \\
$\mathrm{Hb}$ & Before & $12.15 \pm 1.11$ & $13.70 \pm 1.27$ & 0.033 \\
& 2nd week after & $11.05 \pm 1.36$ & $7.40 \pm 1.21$ & 0.129 \\
$\mathrm{TP}$ & Before & $6.75 \pm 0.67$ & $6.85 \pm 0.34$ & 0.075 \\
$\mathrm{Alb}$ & 2nd week after & $6.65 \pm 0.51$ & $4.55 \pm 0.32$ \\
& Before & $4.25 \pm 0.35$ & $4.10 \pm 0.30$ \\
\hline
\end{tabular}

$P O H C$ professional oral health care, $W B C$ white blood cell, $P L T$ platelet, $H b$ hemoglobin, $T P$ total protein, $A l b$ albumin

Assessment of oral environment deterioration

Results of the oral environment assessments, including the OAG score, oral mucositis grade assessment, PCR, Saxon test, and tests with the oral moisture checker (Mucus ${ }^{\mathrm{TM}}$ ) and electrogustometer, are presented in Table 4.

In the POHC group, the OAG score was improved or unchanged in 11 patients, but 1 patient's score worsened during the course of chemotherapy. In the self-care group, five patients experienced an improvement or no change, and nine patients showed deterioration of their OAG score after chemotherapy. There was significantly less deterioration in the POHC group compared with the self-care group $(P=0.01)$.

With regard to the CTCAE grade (oral mucositis grade assessment), none of the patients in the POHC group had grade I or higher oral mucositis. Conversely, in the self-care group, there were three patients with grade I and one patient with grade II oral mucositis. Thus, there were significantly more cases of oral mucositis in the self-care group than in the POHC group $(P=0.04)$ (Table 4$)$.

The PCR showed numerical improvement in 11 patients and deterioration in 1 patient in the POHC group after chemotherapy, as compared with pre-chemotherapy PCR. In the self-care group, six patients showed improvement, while eight patients showed deterioration. Thus, the self-care group showed significantly greater oral health deterioration compared with the POHC group. The Saxon test, oral moisture checker test (Mucus ${ }^{\mathrm{TM}}$ ), and electrogustometer test each demonstrated no statistically significant differences between the two treatment groups with regard to changes before and after chemotherapy (Table 4).

\section{Discussion}

This study, the first of its kind, clearly demonstrated the efficacy of regular POHC on preventing chemotherapyinduced oral mucositis in breast cancer patients. Borowski et al. randomized 166 leukemia patients to receive either professional dental and periodontal care or to receive no professional oral care. They then investigated the incidence of oral mucositis due to chemotherapy and radiotherapy and found that the incidence of oral mucositis in the dental care group was reduced by $70 \%$ compared with that in the control group [23]. In addition, Soga et al. performed a retrospective pilot study in leukemia patients and reported that systematic oral care using a moisturizer reduced the incidence of oral mucositis by $20 \%[24,25]$.

Table 4 Deterioration rates of oral environment assessment parameters $\left(\chi^{2}\right.$ test $)$

\begin{tabular}{llll}
\hline & $\begin{array}{l}\text { Self-care } \% \\
(n=14)(n)\end{array}$ & $\begin{array}{l}\text { POHC \% } \\
(n=12)(n)\end{array}$ & $P$ value \\
\hline OAG & $64.3(9)$ & $8.3(1)$ & 0.005 \\
Oral mucositis grade & $28.6(4)$ & $0.0(0)$ & 0.044 \\
PCR & $57.1(8)$ & $41.7(5)$ & 0.012 \\
Saxon test & $35.7(5)$ & $33.3(4)$ & 0.536 \\
Mucus $^{\text {TM }}$ & $21.4(3)$ & $25.0(3)$ & 0.404 \\
Electrogustometer & $21.4(3)$ & 0.596 \\
\hline
\end{tabular}

$P O H C$ professional oral health care, $P C R$ polymerase chain reaction, $O A G$ Oral Assessment Guide 
The present study was performed in breast cancer outpatients who underwent chemotherapy, a patient population expected to show milder adverse reactions, including oral mucositis, as compared with leukemia patients. However, prior to this study, there have been no reports of oral health care in conjunction with chemotherapy for solid cancers. Since the report by Borowski et al. in 1994, there have been no investigations on the efficacy of $\mathrm{POHC}$ in anticancer therapy consisting only of chemotherapy. In addition, there have been no reports of randomized comparative studies on oral cavity adverse reactions in cancer outpatients undergoing chemotherapy. Since it can be anticipated that the future will see greater numbers of such patients, the importance of conducting clinical studies in these patients is apparent. In Japan, it was reported that only $14 \%$ of hospitals have a dental department, and $20 \%$ of those departments did not perform oral care for patients in other departments [26]. Because the present study demonstrated the importance of POHC performed by dentists and dental hygienists, treating physicians should consider collaboration with family dentists when performing outpatient chemotherapy. Moreover, establishing comprehensive medical teams of dentists, dental hygienists, physicians, pharmacists, nurses, and professionals from other related disciplines will enable the delivery of safer and higher quality medical care to patients, in addition to reducing the individual burden on physicians and nurses [6].

No acute dental infections occurred in either patient group in our study. This may be because, prior to starting chemotherapy, we administered appropriate dental care (including extractions) as necessary in all enrolled patients. On the other hand, while there were no cases of oral mucositis in the POHC group, four patients in the self-care group (28.6\%) developed oral mucositis of grade I or higher severity, which was a statistically significant difference. Significant deterioration of the OAG score, an index of the oral environment, was also seen in the self-care group. In addition, significantly more patients in the self-care group had deterioration of the PCR score, an index of an unclean oral cavity. Katsura et al. performed oral care, centered on moisturization, for dry mouth in patients undergoing radiotherapy for head and neck cancers. In their study, an increase in the decayed, missing, or filled tooth (DMFT) index after 3 years correlated with the timing of intervention, and dry mouth was significantly reduced in patients who had begun oral care prior to radiotherapy [27].

In our study, the results of the Saxon test and the test using the oral moisture-checking device, used as indices of oral dryness, were not significantly different between the POHC and self-care groups. Nevertheless, we believe that it is necessary to perform oral care focused on maintaining oral moisture in order to prevent deterioration of the oral environment due to dryness. Electrogustometry also resulted in no significant differences between the two groups, although the postchemotherapy taste recovery period appeared to be shorter in the POHC group than in the self-care group. However, more detailed studies of these aspects are warranted.

By maintaining the oral environment, $\mathrm{POHC}$ reduces the number of bacteria in the mouth and thereby reduces the incidence of secondary infections of the oral mucosa and periodontal tissues $[1,28-30]$. In our study, it can be surmised that because oral mucositis was prevented and the patients experienced no symptoms, such as pain or bleeding, patients in the control group might have performed adequate self-care. Moreover, we believe that regular assessment of patients' oral cavities by dentists and dental hygienists, followed by strict guidance when deemed necessary, would lead to early discovery of problems such as dry mouth, furry tongue, and mucosal injury due to improper self-care. Suitable countermeasures could then be taken, and this would translate into better maintenance of the oral environment as well as improved patient motivation to perform adequate self-care.

There were certain limitations to this study, including the small sample size. We previously determined that including a minimum of 12 patients in each group would allow for establishing significance; however, additional studies with larger sample sizes are warranted to further confirm our results. Moreover, there was variability in the chemotherapy regimens administered between the POHC and self-care groups. Nevertheless, we believe that our results clearly demonstrate the importance of $\mathrm{POHC}$ in patients receiving chemotherapy.

The results of this study in breast cancer patients receiving adjuvant chemotherapy demonstrated that performance of POHC beginning before the start of chemotherapy significantly reduced both deterioration of the oral environment and the incidence of oral mucositis. These findings confirm the efficacy of regular prophylactic $\mathrm{POHC}$ in cancer patients.

Acknowledgments We thank Ms. H. Fujihira, Ms. R. Baba, and Ms. S. Kojima for help in preparing the manuscript.

Disclosure The authors have no conflicts of interest to declare.

Open Access This article is distributed under the terms of the Creative Commons Attribution Noncommercial License which permits any noncommercial use, distribution, and reproduction in any medium, provided the original author(s) and the source are credited.

\section{References}

1. Sonis ST (1998) Mucositis as a biological process: a new hypothesis for the development of chemotherapy-induced stomatotoxicity. Oral Oncol 34:39-43

2. Majorana A, Schubert MM, Porta F, Ugazio AG, Sapelli PL (2000) Oral complications of pediatric hematopoietic cell transplantation: diagnosis and management. Support Care Cancer 8:353-365

3. Raber-Durlacher JE, Weijl NI, Abu Saris M, de Koning B, Zwinderman AH, Osanto S (2000) Oral mucositis in patients treated with chemotherapy for solid tumors: a retrospective analysis of 150 cases. Support Care Cancer 8:366-371 
4. Barber C, Powell R, Ellis A, Hewett J (2007) Comparing pain control and ability to eat and drink with standard therapy vs Gelclair: a preliminary, double centre, randomised controlled trial on patients with radiotherapy-induced oral mucositis. Support Care Cancer 15: 427-440

5. Sonis ST, Elting LS, Keefe D, Petersen DE, Schubert M, HauerJensen M, Bekele BN, Raber-Durlacher J, Donnelly JP, Rubenstein EB (2004) Mucositis Study Section of the Multinational Association for Supportive Care in Cancer, International Society for Oral Oncology Perspectives on cancer therapy-induced mucosal injury: pathogenesis, measurement, epidemiology, and consequences for patients. Cancer 100:1995-2025

6. Keefe DM, Schubert MM, Elting LS, Sonis ST, Epstein JB, RaberDurlacher JE, Migliorati CA, McGuire DB, Hutchins RD, Peterson DE (2007) Mucositis Study Section of the Multinational Association for Supportive Care in Cancer and the International Society for Oral Oncology Updated clinical practice guidelines for the prevention and treatment of mucositis. Cancer 109:820-831

7. Kennedy HF, Morrison D, Kaufmann ME, Jackson MS, Bagg J, Gibson BE, Gemmell CG, Michie JR (2000) Origins of Staphylococcus epidermidis and Streptococcus oralis causing bacteraemia in a bone marrow transplant patient. J Med Microbiol 49:367-370

8. Yoneyama T, Yoshida M, Matsui T, Sasaki H (1999) Oral care and pneumonia. Oral Care Working Group. Lancet 354:515

9. McGuire DB, Fulton JS, Park J, Brown CG, Correa ME, Eilers J, Elad S, Gibson F, Oberle-Edwards LK, Bowen J, Lalla RV (2013) Mucositis Study Group of the Multinational Association of Supportive Care in Cancer/International Society of Oral Oncology (MASCC/ISOO) Systematic review of basic oral care for the management of oral mucositis in cancer patients. Support Care Cancer 21: 3165-3177

10. Greene FL, Page DL, Fleming ID, Fritz AG, Balch CM, Haller DG, Morrow M (2002) AJCC cancer staging manual, 6th edn. Springer, New York

11. Common terminology criteria for adverse events v3.0 (CTCAE) (2006) US National Cancer Institute. http://www.eortc.be/services/ doc/ctc/ctcaev3.pdf. Accessed 23 Jan 2013

12. Eilers J, Berger AM, Peterson MC (1988) Development, testing, and application of the oral assessment guide. Oncol Nurs Forum 15:325330

13. Koshino M, Sakai C, Ogura T, Kawasaki A, Fukuzato F, Miyazaki Y (2009) Efficacy of oral cavity care in preventing stomatitis (mucositis) in cancer chemotherapy. Gan To Kagaku Ryoho 36: $447-451$

14. O'Leary TJ, Drake DB, Naylor JE (1972) The plaque control record. J Periodontol 43:38

15. Seki S, Sonoda S, Suzuki M, Fujii W (2003) The saliva secretion test in stroke patients: reliability of the Saxon test. Japan J Rehabil Med 40:858-862

16. Jellema AP, Slotman BJ, Doornaert P, Leemans CR, Langendijk JA (2007) Impact of radiation-induced xerostomia on quality of life after primary radiotherapy among patients with head and neck cancer. Int $\mathrm{J}$ Radiat Oncol Biol Phys 69:751-760

17. Tamura Y, Sakurai A, Yamamura C (2009) Comparison of oral mucosa humidity and salivary flow rate, and the effects of moisturizer on the salivary flow rate. Niigata Dent J 39:35-42
18. Hovan AJ, Williams PM, Stevenson-Moore P, Wahlin YB, Ohrn KE, Elting LS, Spijkervet FK, Brennan MT (2010) Dysgeusia Section, Oral Care Study Group, Multinational Association of Supportive Care in Cancer (MASCC)/International Society of Oral Oncology (ISOO) A systematic review of dysgeusia induced by cancer therapies. Support Care Cancer 18:1081-1087

19. Kobayashi M, Okada Y, Toda K (2007) Gustatory sensitivity in different regions of the tongue in Japanese young women. J Jpn Dent Soc Orient Med 25:1-2

20. Schubert MM, Williams BE, Lloid ME, Donaldson G, Chapko MK (1992) Clinical assessment scale for the rating of oral mucosal changes associated with bone marrow transplantation. Development of an oral mucositis index. Cancer 69:2469-2477

21. Sonis ST, Eilers JP, Epstein JB, LeVeque FG, Liggett WH Jr, Mulagha MT, Peterson DE, Rose AH, Schubert MM, Spijkervet FK, Wittes JP (1999) Validation of a new scoring system for the assessment of clinical trial research of oral mucositis induced by radiation or chemotherapy. Mucositis Study Group. Cancer 85: 2103-2113

22. McGuire DB, Peterson DE, Muller S, Owen DC, Slemmons MF, Schubert MM (2002) The 20 item oral mucositis index: reliability and validity in bone marrow and stem cell transplant patients. Cancer Investig 20:893-903

23. Borowski B, Benhamou E, Pico JL, Laplanche A, Margainaud JP, Hayat M (1994) Prevention of oral mucositis in patients treated with high-dose chemotherapy and bone marrow transplantation: a randomised controlled trial comparing two protocols of dental care. Eur J Cancer B Oral Oncol 30B:93-97

24. Sugiura Y, Soga Y, Tanimoto I, Kokeguchi S, Nishide S, Kono K, Takahashi K, Fujii N, Ishimaru F, Tanimoto M, Yamabe K, Tsutani S, Nishimura F, Takashiba S (2008) Antimicrobial effects of the saliva substitute, oral balance, against microorganisms from oral mucosa in the hematopoietic cell transplantation period. Support Care Cancer 16:421-424

25. Soga Y, Sugiura Y, Takahashi K, Nishimoto H, Maeda Y, Tanimoto M, Takashiba S (2010) Progress of oral care and reduction of oral mucositis - a pilot study in a hematopoietic stem cell transplantation ward. Support Care Cancer 19:303-307

26. Japanese Society of Oral and Maxillofacial Surgeons (2011) Report of survey involving hospital oral and maxillofacial surgery. http:// www.jsoms.or.jp/sns/uploads/fckeditor/file/enquete/H19Hospital/ H19enquete2.pdf

27. Katsura K, Goto S, Sasai K, Sato K, Tomita M, Matsuyama H, Hayashi T (2009) The effect of dental management for maintaining dental health in patients with head and neck cancer after radiotherapy. Japan J Head Neck Cancer 35:266-272

28. Akintoye SO, Brennan MT, Graber CJ, McKinney BE, Rams TE, Barrett AJ, Atkinson JC (2002) A retrospective investigation of advanced periodontal disease as a risk factor for septicemia in hematopoietic stem cell and bone marrow transplant recipients. Oral Surg Oral Med Oral Pathol Oral Radiol Endod 94:581-588

29. Raber-Durlacher JE, Epstein JB, Raber J, van Dissel JT, van Winkelhoff AJ, Guiot HF, van der Velden U (2002) Periodontal infection in cancer patients treated with high-dose chemotherapy. Support Care Cancer 10:466-473

30. Myers RA, Marx RE (1990) Use of hyperbaric oxygen in postradiation head and neck surgery. NCI Monogr 9:151-157 